\title{
El Romanticismo alemán y el drama
}

\author{
[German Romanticism and Drama] \\ http://dx.doi.org/10.11606/1982-88372545110
}

Miguel Alberti ${ }^{1}$

\begin{abstract}
German Romanticism made use of drama less frequently and less successfully than the aesthetic movements that preceded it. However, there was a German Romantic theater, and it developed a number of distinctive characteristics. As these features are analyzed in the light of the Romantic program itself, one can see with rather greater clarity some of the possible reasons why the German Romantics not only failed to produce a large number of plays, but also did not find in drama an appropriate vehicle for an important part of their agenda. This paper carries out such an analysis and reflects on the nature of German Romantic drama and the reasons for its comparatively minor development.
\end{abstract}

Keywords: German Romanticism; "Universal Drama”; Romantic Irony.

Resumen: El Romanticismo alemán se sirvió del drama con menos frecuencia y con menos éxito que los movimientos estéticos que lo precedieron. Pero existió un teatro romántico alemán y llegó a desarrollar una serie de características distintivas. En la medida en que estas características se analizan a la luz del programa del Romanticismo mismo, se pueden ver con algo más de claridad algunas de las posibles razones por las cuales los románticos alemanes no solo no produjeron gran cantidad de obras teatrales sino que tampoco encontraron en el teatro un vehículo apropiado para parte importante de su propuesta. El objetivo del presente artículo es llevar a cabo dicho análisis y reflexionar en torno a la naturaleza del drama alemán del Romanticismo y las razones de su desarrollo comparativamente menor.

Palabras clave: Romanticismo alemán; “drama universal”; ironía romántica.

\section{Introducción}

"Las preguntas son el comienzo de todo saber. Si ha de hablarse con seriedad de 'drama romántico', el concepto deberá tolerar, entonces, la pregunta acerca de si tal cosa en general existe". Con esta reflexión inicia Gerhard Schulz el escrito introductorio de la compilación sobre teatro del Romanticismo alemán Das romantische Drama (JAPP et. al. 2000: 1 ). ${ }^{2}$ Por supuesto que, en principio, podría imponerse la misma reserva (“ ¿existe

\footnotetext{
${ }^{1}$ Universidad Nacional de Mar del Plata, Av. Funes, 3350, Mar del Plata, Buenos Aires, 7600, Argentina. E-mail: alberti.miguel@gmail.com. ORCID: 0000-0002-4276-2770

${ }^{2}$ Cuando se utiliza una traducción ajena, es la que se indica en el lugar correspondiente de las "Referencias bibliográficas". En los casos en los que no se menciona allí una edición en español (generalmente, cuando el texto no está traducido), la versión castellana es propia.
}

\section{(cc) BY-NC}

Pandaemonium, São Paulo, v. 25, n. 45, jan.- abr. 2022, p. 110-135 
ALBERTI, M. - El Romantismo Alemán y el Drama

realmente este objeto de análisis que pretendemos abordar?") a un estudio sobre cualquier asunto. Y, sin embargo, no es un reparo que aparezca con tanta frecuencia cuando se trata de otros períodos del drama alemán ni cuando se trata de otros géneros dentro del Romanticismo. Si se fuera a abordar la novela romántica, o el fragmento romántico, o bien el drama de la Ilustración o el del Sturm und Drang, la cuestión acerca de la entidad del tema mismo estaría ya zanjada antes de ser siquiera propuesta. Claro que siempre será posible cuestionar lo estrictamente "romántico" del objeto de estudio: los límites de lo que puede o debe ser considerado stricto sensu "Romanticismo" son reconocidamente inciertos (asunto al que se aludirá en el próximo apartado). De todos modos, una vez concedido que existe el Romanticismo en general (se tome el término en un sentido más estrecho o en uno más expandido), sería poco probable que se planteara una duda, por ejemplo, acerca de la existencia misma de la novela romántica. ¿Qué duda cabe en torno a la existencia de la novela romántica alemana? Podemos nombrar novelas destacadas y mundialmente reconocidas de casi cada uno de los autores que vinculamos, sin mayores cuestionamientos, al Romanticismo alemán. Respecto del drama romántico, en cambio, la pregunta surge toda vez que aspiramos a hablar "con precisión” del tema: ¿existe realmente el teatro romántico?; ¿qué es exactamente, en caso de que exista, el teatro romántico alemán? Es tan sintomático el hecho de que en historias del drama alemán esté ausente (o casi) el Romanticismo como el de que en presentaciones sobre el Romanticismo alemán esté ausente (o casi) el drama. ${ }^{3}$

La pregunta por la entidad del teatro del Romanticismo incomoda inmediatamente de dos modos. Por un lado, en este caso se torna mucho más indispensable que en otras

\footnotetext{
${ }^{3}$ El primer volumen de Das deutsche Drama de Benno von Wiese (1968), una compilación canónica de estudios sobre teatro alemán, pasa de Lenz (representante del Sturm und Drang) a obras en general de madurez de Schiller y Goethe, de allí a Kleist (un autor que en las periodizaciones académicas cae por fuera de lo que se considera Romanticismo en sentido estricto) y de allí ya a Grillparzer. El editor sostiene que en su selección "ha intentado destacar ejemplos representativos que, en el marco del drama alemán, hayan tenido una importancia particular en cuanto a la creación de estilos o desde el punto de vista de la historia de las ideas" (1968: 5). No habría ejemplos de esto, entonces, en el drama del Romanticismo. Es similar el caso bastante más cercano en el tiempo de la compilación hecha por Harro Müller-Michaels (1985: x), cuyos criterios de selección de autores y obras fueron la "historicidad" (que hayan significado un aporte novedoso en su época) y la "actualidad" (que contuvieran aportes de significación para la discusión de "problemas actuales"). Tampoco incluye autores románticos. Por el otro lado, en las investigaciones sobre el Romanticismo se le suele prestar poca atención al drama, cuestión que, según la reconstrucción de Stefan Scherer en un capítulo muy valioso sobre el drama romántico, reposa fundamentalmente en el hecho de que la reunión de géneros característica del programa romántico (sobre la que se hablará más adelante) se materializaba mejor en la novela y a que el valor de los dramas románticos era más bien bajo y que por su naturaleza tendían a producir, más bien, aburrimiento. Sin embargo, como Scherer mismo señala, es posible que el rechazo de este tipo nuevo de drama y el impacto negativo que producía -que a su vez generó que se le prestase menos atención- se hayan debido precisamente a lo que tiene de más innovador, a su ruptura con las convenciones del género (2003: 79-80).
}

Pandaemonium, São Paulo, v. 25, n. 45, jan.- abr. 2022, p. 110-135 
ALBERTI, M. - El Romantismo Alemán y el Drama

ocasiones definir con cierta precisión a qué llamaremos drama romántico. A varios dramaturgos (los dos casos más evidentes son el de Schiller y - más aún - el de Kleist) los vinculamos cotidianamente, y no sin razones atendibles, a lo que popularmente llamamos "Romanticismo alemán” en un sentido amplio. Sin embargo, y con sobrados argumentos, en el ámbito académico casi nunca son alineados con el movimiento mismo del Romanticismo alemán, ya que no pertenecen a lo que en términos globales se denomina, desde la famosa obra de Heine (1961), "la escuela romántica", 4 y porque sus obras carecen de mucho de lo que se considera más característico de la estética del Romanticismo propiamente dicho. De modo tal que el asunto se plantea de la manera siguiente: hay teatro durante la época de florecimiento del Romanticismo; hay, de hecho, dramaturgos que comparten rasgos y tendencias con los románticos; pero ¿hay un teatro que podamos llamar romántico con pleno derecho?; ¿hay autores indiscutiblemente románticos a los que podamos calificar con justicia de "dramaturgos"? Una vez que se estrecha el círculo de esta manera surge la segunda dificultad: a primera vista resulta bastante poco claro (mucho menos claro que en el caso de la novela) cuáles serían grandes autores o grandes obras de teatro romántico. ¿Hay mucho más que El gato con botas y los otros dramas más o menos conocidos de Tieck? ¿Hay ejemplos de importancia de teatro romántico? ¿Los suficientes como para que hablemos de "drama romántico" con una soltura equivalente a la que nos podemos permitir al hablar de "novela romántica"?

A todo esto se suma un último y fundamental problema: concedido que hay obras dramáticas escritas por autores que podemos considerar sin conflicto románticos, ¿son, estas obras, románticas ellas mismas? Dicho de otro modo: ¿coinciden con la poética romántica en general?; ¿representan el programa o las notas típicas del Romanticismo alemán? En ese caso, ¿cuáles serían los rasgos propiamente definitorios del teatro romántico? La respuesta de esta última pregunta será el objetivo de máxima del presente artículo, y a este propósito fundamental precederá un abordaje de algunas de las preguntas anteriores. Concretamente: un comentario sobre (1) la especificidad del término “romántico" en el contexto alemán en torno al año 1800; (2) la existencia de un auténtico teatro romántico; (3) las posibles razones del lugar menos protagónico que ocupó el teatro en el período romántico de la literatura alemana.

\footnotetext{
${ }^{4}$ Con posterioridad, el rótulo (aunque no carece de arbitrariedad) sería reutilizado y consagrado por uno de los estudios clásicos más valorados en la investigación sobre el Romanticismo alemán: Die romantische Schule (1870) de Rudolf Haym.
}

Pandaemonium, São Paulo, v. 25, n. 45, jan.- abr. 2022, p. 110-135 


\section{Vacilaciones generales en torno al "Romanticismo alemán" y circunscripción del objeto de estudio}

Es moneda corriente en estudios sobre el Romanticismo alemán, o sobre el Romanticismo en general, la afirmación de que se trata de una cuestión de límites difusos y particularmente pantanosa. Paul Kluckhohn, un intelectual que aportó mucho al estudio serio y cuidadoso del Romanticismo alemán, empezó su libro Das Ideengut der deutschen Romantik (1942: 1) afirmando que "pocos conceptos de la historia de la poesía y el espíritu alemanes son tan controvertidos y ambiguos como el concepto "romanticismo"”. Hace tres años, tres cuartos de siglo después del libro de Kluckhohn, Helmut Schanze empieza el suyo, Erfindung der Romantik, diciendo que "apenas si existe un rótulo para una época acerca del cual se haya disputado con tanta insistencia y hasta hoy como se disputa en torno al concepto de 'Romanticismo'” (2018: 1). Ya en 1836, aún en época de vigencia plena del Romanticismo, Alfred de Musset se reía, en la primera de sus Lettres de Dupuis et Cotonet (1888), del carácter prácticamente indescifrable del concepto de lo romántico y acababa por concluir que el Romanticismo no consiste más que en la sobreabundancia de adjetivaciones. Bastante tiempo después, un famoso artículo de Arthur Lovejoy ("On the Discrimination of Romanticisms", de 1924) plantearía el asunto de modo polémico en el ámbito académico sosteniendo que la entidad del Romanticismo en tanto tal debía ser puesta en duda, puesto que no era posible identificar suficientes rasgos característicos que permitieran hablar con propiedad de un auténtico "movimiento". En el mejor de los casos podría hablarse de "romanticismos" en plural. La respuesta, dos décadas y media después, de René Wellek (“The Concept of Romanticism in Literary History”, de 1949), intentaría aportar fundamentos a la idea de que sí existen determinados tópicos o concepciones básicas que están presentes en los distintos romanticismos, es decir: en el Romanticismo en general. Este artículo daría origen a lo que se conoce como el debate "Lovejoy-Wellek", retomado posteriormente por autores como Jerome McGann (1985) o Robert Griffin (1995); ${ }^{5}$ un debate que no solo importa en función de la pregunta por la(s) naturaleza(s) del Romanticismo sino también en función de preguntas de mayor espectro (como qué elementos deben ser evaluados en general para adjudicar unidad a distintas obras, a distintos autores, etc.). En fin: se trata de una cuestión

\footnotetext{
${ }^{5}$ Debo mucho de esta reconstrucción de los debates en torno a la “definición” del Romanticismo a Jerónimo Ledesma, de la Universidad de Buenos Aires.
}

Pandaemonium, São Paulo, v. 25, n. 45, jan.- abr. 2022, p. 110-135 
ALBERTI, M. - El Romantismo Alemán y el Drama

compleja y muy difícil de dirimir, que nació y se consolidó ya en la primera mitad del siglo XIX y que sigue teniendo vigencia hoy.

En el caso específico del Romanticismo alemán se podrían plantear dificultades equivalentes. Hay un punto mucho más allanado, que obedece a que hay un considerable consenso respecto de que se trata de "romanticismos alemanes" en plural. Desde hace más de medio siglo la crítica se acostumbró a hablar, por ejemplo, de "romanticismo temprano" ("Frühromantik") o "más antiguo" (“Ältere Romantik"), también señalado como "romanticismo de Jena"; en segundo lugar, hablamos de "romanticismo más joven" (“Jüngere Romantik"), "alto romanticismo" ("Hochromantik”) o "romanticismo medio" ("Mittlere Romantik"), también "romanticismo de Heidelberg"; y por último de "romanticismo tardío" ("Spätromantik") para la franja más demorada y dispersa del movimiento en términos locales y espaciales. En ocasiones esta tendencia a diferenciar entre las distintas manifestaciones del Romanticismo alemán llegó a negar la existencia misma del movimiento en tanto tal, como en el caso de Schulz (1983: 76): "no existe un Romanticismo en tanto un período que se desarrolle de acuerdo a un programa estético o un principio fundamental estético, y menos aún existen los románticos". Sin embargo, qué deba y quiénes deban formar parte de este conjunto de romanticismos alemanes no es algo exento de polémica o, al menos, de confusión. Un caso evidente es el ya señalado de Heinrich von Kleist, aunque este es solo uno entre varios personajes que si bien se acercan en distintos puntos al Romanticismo (y que fuera de la academia tendemos a asociar a él) no se inscriben con pleno derecho en el movimiento, como Friedrich Hölderlin o Jean Paul, o, bastante más alejados, Schiller o incluso Goethe (quien habría visto con bastante desagrado, por distintas razones, que se lo agrupara bajo el rótulo de "Romanticismo alemán"), respecto de quien dice Schulz, en la introducción ya mencionada a Das romantische Drama, que bien podría reconocérsele una cierta "romanticidad", "como ocurre en la investigación literaria no alemana y como ya lo habían hecho los hermanos Schlegel” (2000: 17, cf. también HINDERER 2002, KREMER; KILCHER 2015, etc.).

La indefinición del asunto parece apoyarse en el hecho de que los diversos elementos que vinculamos al Romanticismo son lo suficientemente generales, vagos y abundantes como para que varios de ellos se encuentren presentes en multitud de autores, en multitud de obras, etc. Aun así, si queremos hablar del "Romanticismo alemán", y, en particular, del teatro del Romanticismo alemán, habrá que estrechar el círculo. A estos 
ALBERTI, M. - El Romantismo Alemán y el Drama

fines resulta cómoda, como primer paso, la división que articula el difundido manual de Safranski (2007) entre "el Romanticismo" (el movimiento mismo) y "lo romántico” en tanto cosmovisión o ideología que, además de encontrarse presente en las obras de los románticos, se manifiesta también en un campo que es muchísimo más amplio, sobre todo en cuanto a la época y en cuanto al alcance (no se reduce al mundo literario, ni siquiera al artístico en general, sino que está activo y se manifiesta en movimientos sociales, estudiantiles, políticos, etc.). Como definición del Romanticismo propiamente dicho, en tanto programa estético, podrían valer con igual o similar derecho propuestas entre sí bastante dispares, según el rasgo que se decida poner en primer plano o el movimiento al que se decida contraponerlo. Kremer y Kilcher, con auténtico espíritu de manual (en el mejor sentido), encuentran una solución tan cómoda como inobjetable al definir al Romanticismo, en tanto teoría estética, como una "teoría de la imaginación y lo fantástico" que se contrapone esencial e integralmente a "los conceptos ilustrados de imitación de la naturaleza y de instrucción moral” (2015: 44). ${ }^{6}$

Dentro de lo que llamamos "Romanticismo" en sentido más o menos "técnico" cabe hacer aquí, todavía, una especificación más, que afecta en particular al objetivo de este artículo. En lo que sigue se tratará fundamentalmente de lo que pudiera ser llamado "drama romántico" con la mayor propiedad posible, es decir, de dramas compuestos por escritores cuya inscripción en el movimiento es de aceptación generalizada y que, además, pueden ser considerados expresiones características de la poética romántica en general. Esta restricción reduce muy considerablemente el objeto de estudio. Fuera de algunas alusiones de carácter general a autores del movimiento romántico, el rastreo de elementos definitorios del drama del Romanticismo alemán a continuación se hará fundamentalmente a partir de la obra de Ludwig Tieck, el único que formó parte de la Frühromantik o "Romanticismo temprano", es decir, de la fase fundacional del movimiento, que fue, a su vez, la fase más involucrada con la generación y puesta en marcha de un programa literario. Por lo demás, los de Tieck siguen siendo hoy los más estudiados, traducidos y valorados de los dramas románticos. ${ }^{7}$ Esto no obstante, a

\footnotetext{
${ }^{6}$ Una reconstrucción muy sintética y útil de la recepción en general del Romanticismo alemán en el ámbito de la investigación académica en Alemania hasta fines del siglo pasado puede encontrarse en la sección final del libro de Detlef Kremer Prosa der Romantik (1997: 175-185).

${ }^{7}$ En el caso de Tieck (como en tantos otros) resulta difícil dar con presentaciones introductorias a la vez abarcadoras y rigurosas en castellano. Puede ser útil para una visión sinóptica el estudio de Víctor Grovas (2001) El mundo al revés y la sonrisa romántica, dedicado íntegramente a su comedia.
} 
ALBERTI, M. - El Romantismo Alemán y el Drama

continuación se harán algunas aclaraciones de mayor alcance destinadas a aportar un marco de orden más general.

\section{Presencia y ausencia del drama en el Romanticismo alemán}

De August Wilhelm Schlegel conservamos un único drama (Jon) y de su hermano Friedrich otro (Alarkos), ambos presentados en 1802, ninguno de significación en sus respectivas obras completas, ninguno una auténtica puesta en práctica de los principios estéticos del Romanticismo, ni siquiera, podría decirse, de sus propias teorías sobre el drama; de Friedrich von Hardenberg (Novalis), no conservamos ninguno; Wilhelm Heinrich Wackenroder tampoco compuso obras teatrales; tampoco se inclinó al teatro E.T.A. Hoffmann; ni Wilhelm Hauff... Si queremos encontrar obras de teatro de cierta significación en autores románticos tenemos que apuntar a Achim von Arnim, a Clemens Brentano, a Joseph von Eichendorff, a Friedrich de la Motte Fouqué y - como se señaló, el más estudiado en tanto dramaturgo - a Ludwig Tieck. ${ }^{8}$ Ahora bien: con la excepción de Fouqué, que produjo una muy abultada obra dramática que además tendría un impacto directo e importantes proyecciones en obras de arte alemanas posteriores (tema que se tocará en el apartado siguiente), ninguno de estos últimos autores nos dejó más dramas que narraciones y en general a ninguno lo conocemos principalmente como dramaturgo.

Se trata de un punto que amerita una aclaración de importancia: estas afirmaciones acerca del predominio de la producción narrativa por sobre la dramática en los autores románticos que sí compusieron obras de teatro no pueden ser generalizadas y proyectadas sobre la época como un todo, como si se tratara de un fenómeno ampliamente difundido. No encontramos este predominio marcado de la narración en Goethe, ni en Schiller, ni en Kleist. Mucho menos en los autores más nombrados de la Ilustración o, para el caso, del Sturm und Drang. Todo esto equivale a decir que el del Romanticismo es, en este plano, un caso aparte. A fines del siglo XVIII se apelaba al teatro como medio expresivo, de hecho, desde puntos de vista estéticos que se pensaban a sí mismos como contrapuestos.

Quizás debamos inferir, entonces, que si el Romanticismo tomó distancia del escenario fue porque "decidió" hacerlo. Por supuesto, esto no implica suponer algo tan indefendible como que el Romanticismo veía como insuficiente al discurso dramático en

\footnotetext{
${ }^{8}$ Esta lista de autores, coincidente con el breve pero denso texto de Stockinger (2004), reduce la selección a los "indiscutibles": otras listas pueden encontrarse sintetizadas y comentadas (junto con algunas interesantes categorizaciones generales sobre la comedia romántica) en Japp (1999: 9-15).
}

Pandaemonium, São Paulo, v. 25, n. 45, jan.- abr. 2022, p. 110-135 
ALBERTI, M. - El Romantismo Alemán y el Drama

tanto tal: baste recordar que a August Wilhelm Schlegel (que también tradujo a Calderón) y a Ludwig Tieck (que también tradujo el Quijote) se debe la destacada edición en nueve volúmenes de los Shakespeare's dramatische Werke. Por lo demás, la escasez de producción dramática no va de la mano de una escasez correlativa de teoría sobre el drama de parte de estos autores. Bien por el contrario, el drama está muy presente y con un rol destacado en la teoría de Schlegel, y aparece en autores del mayor grado de distanciamiento posible respecto del drama (en cuanto a su propia producción), como en Novalis. ${ }^{9}$

Sí podría plantearse la pregunta por el alejamiento del teatro en la práctica misma de los románticos. Es decir, que quizás había algo en el programa romántico mismo que favorecía que se renunciara (parcialmente) al medio expresivo de la época por excelencia. Y aquí cabe hacer una aclaración de importancia: esta renuncia, como se señalará con algo más de detalle en el próximo apartado, no solo se materializa en una escasez de producción dramática de parte de los románticos en términos generales sino que también es visible en la obra dramática que los románticos sí produjeron, puesto que en reiteradas ocasiones esta obra dramática no estaba pensada para ser representada sino en cambio para ser leída, para ser apreciada como una obra literaria ajena a la realidad artística y social del escenario.

Para intentar dar respuesta a esta cuestión de las posibles motivaciones del Romanticismo para prescindir del mecanismo expresivo de mayor vigencia en su época se podría apelar a toda una serie de especulaciones cuyo grado de verosimilitud, en muchos casos, dependerá del que decidamos otorgarle. Aquí quisiera repasar únicamente unos pocos puntos que hacen a lo más nuclear del programa romántico y que quizás pueden aportar algún indicio en la búsqueda de una explicación para el vínculo nuevo y propio que sostuvo el Romanticismo alemán con el teatro.

\footnotetext{
${ }^{9}$ En el texto de Michele Cometa (2000) se llega a hacer del teatro una especie de hilo conductor subterráneo de la obra teórica en general de Friedrich Schlegel, "el padre filosófico del romanticismo" (2000: 21). Es cierto que la práctica inexistencia de obra dramática en Schlegel no se contradice para nada con su interés por el teatro, que lo acompañó durante muy extensos períodos de su producción. Para las reflexiones sobre el drama de Novalis véase el texto (2000) de Johannes Endres.
} 


\section{Entidad del teatro alemán en la segunda mitad del siglo XVIII}

Prodesse et delectare, "instruir y agradar", es reconocidamente la máxima capital de la poética de la Ilustración. ${ }^{10}$ Por cierto, instruir y agradar a todos, ya que todos, en completo rigor filosófico, somos miembros en igual medida y con igual derecho del único conjunto que nos diferencia de algún otro: todos somos miembros iguales de la Humanidad. Y todos somos capaces de ser instruidos y de sentir agrado ante una obra de arte construida de manera acertada.

El rey de la Ilustración racionalista "alemana" (las comillas obedecen a que su propia pretensión explícita era la imitación sistemática de modelos extranjeros), Johann Christoph Gottsched, sostenía que una buena obra literaria se compone siguiendo la siguiente receta:

En primer lugar elíjase un instructivo tema moral, que habrá de ser soporte de toda la composición, de acuerdo a la naturaleza del objetivo que se persigue. A estos fines idéese una circunstancia completamente corriente en la que se presente una acción, de modo tal que esta doctrina elegida alcance de manera patentísima a los sentidos. (1968: 214)

A la hora de la ejecución de este plan, el dramaturgo contaba con la ayuda inestimable de la ya bi-milenaria tradición de las tres reglas de unidad aristotélicas, a las que se sumaban cláusulas y restricciones que permitían conservar el decoro o la división de géneros teatrales en función del estamento social al que pertenecían los personajes y demás convenciones.

La reacción contra este formato encorsetado nació ya en el seno de la Ilustración misma (Lessing cuenta, a la vez, como uno de los mejores detractores de Gottsched por un lado y como una de las cúspides del drama de la Ilustración por el otro), pero se hizo consigna y bandera con el breve brote de Sturm und Drang en, sobre todo, la década de 1770. Esta reacción se debía a un rechazo de orden eminentemente formal: el Sturm und Drang reacciona contra la estética de la Ilustración y no necesariamente contra su espíritu en general. Advertir que se podía hacer teatro sin respetar las reglas de unidad, aprendizaje que los jóvenes del Sturm und Drang parecen haber debido a Shakespeare, produjo un efecto liberador muy potente (basta ver el conocido discurso del joven Goethe "En el día de Shakespeare" [2000] para hacerse una idea de lo revelador que le resultó el encuentro con su obra). Un efecto, en verdad, realmente desatado: la acción de Götz von

\footnotetext{
${ }^{10}$ Para una presentación integral de las teorías sobre el teatro en toda esta época de la segunda mitad del siglo XVIII y la primera del XIX puede consultarse en castellano la introducción (y los textos mismos) de la antología de Regula Rohland y Miguel Vedda (2004) La teoría del drama en Alemania (1730-1850).
}

Pandaemonium, São Paulo, v. 25, n. 45, jan.- abr. 2022, p. 110-135 
ALBERTI, M. - El Romantismo Alemán y el Drama

Berlichingen - la primera gran obra de teatro de Goethe, de 1773 [1957] - cambia de lugar más de cincuenta veces, transcurre a lo largo de un extenso período de tiempo, modifica varias veces el núcleo de la acción, adopta un lenguaje coloquial y hasta grosero, se ocupa de campesinos y del Emperador en igual medida... En suma, hace un auténtico esfuerzo (pensemos en las dificultades que los cambios escénicos habían de ocasionar a la hora de presentar la obra en una sala de teatro) para echar por tierra cada una de las cláusulas formales convencionales defendidas y puestas en marcha por el teatro del siglo XVIII.

Un cuarto de siglo después, en la época de gestación del Romanticismo sobre fines del siglo XVIII, la posibilidad de ignorar las normas del teatro de la Ilustración ya era terreno ganado para el artista y los desafíos habían pasado a ser otros. El teatro, hasta cierto punto, ya se había emancipado, ya había conquistado una forma propia de lo que Kant llamaría (en época coincidente con el declive del Sturm und Drang) "mayoría de edad” en su respuesta a la pregunta “¿qué es la Ilustración?”. La estética ilustrada ya no representaba un enemigo amenazante al acecho ante cualquier irreverencia de parte de los artistas; más bien lo contrario: se había transformado paulatinamente en una figura bufonesca y pasada de época.

Sin embargo, el Romanticismo tenía un conflicto de otro orden con el pasado reciente; un choque que superaba muy ampliamente las fronteras de la estética (o las extendía hasta regiones mucho más distantes). Este conflicto de orden más global surgió fuera del escenario y se materializó y desplegó también en otros ámbitos. No es, insistamos, un distanciamiento de orden exclusiva ni prioritariamente estético: más bien se trata de que toda la cosmovisión ilustrada se le presentaba al Romanticismo como una suerte de estafa a la maravilla del mundo, a la potencia del Yo, a la divinidad, al sentimiento. El mundo "exageradamente" secularizado, en el que la Crítica kantiana había a la vez cristalizado un modo de pensar contemporáneo y dado fundamento a un espíritu de renuncia al contacto con la trascendencia, resultaba demasiado vacío y desprovisto de encanto para una generación de jóvenes que había vivido la Revolución Francesa teniendo entre catorce (Schelling) y veintiún años (A.W. Schlegel). Podría decirse que el desafío que tenían los primeros románticos no era tanto el de liberar de ataduras al arte sino más bien el de liberar de ataduras al espíritu (el arte sería el vehículo para este fin más elevado). 
ALBERTI, M. - El Romantismo Alemán y el Drama

No obstante, todos entendían lo suficiente de filosofía como para saber que los logros de la Ilustración no debían ser impugnados o ignorados sin más. Todos percibían la firmeza (y la pertinencia) del edificio kantiano. La crítica le había puesto unos límites al conocimiento que no podían ser desatendidos: pretender formar algo nuevo, más profundo y ambicioso, más libre y desatado, con las mismas herramientas y con los mismos materiales con los que se había construido esta estructura era un emprendimiento destinado al fracaso. La vía para salir de los techos bajos y los ambientes estrechos y opresivos del edificio racionalista debía abrirse en otro lado.

"Por cierto, todo esto deberá ser vertido en otro lenguaje", se anota Novalis en uno de sus apuntes filosóficos conocidos como Fichte-Studien de los años 1795-1796 (2007: 160), ${ }^{11}$ y la frase podría valer como consigna del "romanticismo temprano" en general. Hacía falta un nuevo lenguaje, uno que pudiera sondear con recursos propios los espacios ilimitados que se extendían por fuera del edificio de la razón. Un lenguaje que no se limitase a sistematizar una realidad común a todos o, siguiendo la máxima ilustrada (heredada de una lejana tradición), a "imitar lo bello" de la naturaleza. Al contrario: se trataba de desarrollar un lenguaje capaz de percibir singularidades y de servir como vehículo para la expresión de un Yo libre, expansivo, poderoso y creador.

Desde temprano se identificó ese lenguaje con el del arte, y, en particular, con el de la "poesía" (en un sentido muy amplio del término). Esta poesía, que abarca desde el poema hasta la novela o incluso hasta esferas menos definidamente vinculadas a lo literario; una poesía que en esencia es, en tanto póiesis, "creación", "producción”, no puede, claro está, pretender demostrar nada: la demostración, en los ámbitos en los que es posible que se dé, es privilegio de la filosofía. Pero quizás le sea posible a la Poesie romántica mostrar lo que la filosofía no podría demostrar jamás. Aceptado que la razón tiene límites y que el lenguaje de la razón, la filosofía, comparte con ella idénticas limitaciones, quedaba por ver si otra vía (quizás esta otra vía de la práctica "poética") podía dar cuenta, de algún modo, de aquello que se encuentra más allá de esos límites, es decir, dar cuenta de lo que en esta época de fines del siglo XVIII pasó a llamarse en términos generales "lo absoluto" ("das Absolute").

Claro está que esta posible "mostración" de lo absoluto siempre iba a ser una mostración "a medias", deficitaria desde el punto de vista del conocimiento. Y aquí está

\footnotetext{
${ }^{11}$ En las citas de Novalis se indica la referencia a la traducción (cuando se sigue una traducción publicada) o la referencia a la edición de sus obras completas indicada en las "Referencias bibliográficas", con romanos para el volumen y arábigos para la página.
}

Pandaemonium, São Paulo, v. 25, n. 45, jan.- abr. 2022, p. 110-135 
ALBERTI, M. - El Romantismo Alemán y el Drama

una de las claves del proyecto romántico (aspecto que tendrá un despliegue fundamental en la obra dramática de sus representantes): lo mejor que se puede hacer, en principio, con la imposibilidad de conocer lo absoluto es ponerla en evidencia; es exhibir una y otra vez que se tiene consciencia de la propia limitación; mostrar, por así decir, que se sabe que no se sabe. Una metodología que remite de manera directa y evidente (y consciente) a la ironía socrática. Sócrates era superior a sus conciudadanos porque tenía la sabiduría suficiente como para saber (a diferencia de ellos) que no sabía. Así como aquí el vínculo más cercano con la sabiduría viene dado por la afirmación voluntaria de ignorancia, será la renuncia voluntaria a lo absoluto lo que dé al programa del primer romanticismo lo más cercano posible, en una primera instancia, a un contacto de algún tipo (en cualquier caso no congnoscitivo) con ese plano de lo absoluto mismo. Entre los apuntes de Novalis, por ejemplo, encontramos esta cita:

Mediante la libre renuncia al absoluto surge en nosotros la actividad libre e infinita - el único absoluto posible que nos puede ser dado y que encontramos a causa de nuestra incapacidad de alcanzar y conocer un absoluto. Este absoluto que nos es dado solo puede ser conocido negativamente en la medida en que actuamos y descubrimos que ninguna acción alcanza lo que buscamos. (2007: 173)

En esto mismo consiste, a su vez, la forma de ironía con la que está vinculado muy estrechamente el movimiento y que reaparecerá en su producción dramática: la "ironía romántica". Esta ironía, difícil de definir de un modo tal que cubra el ancho campo de su polisemia, ${ }^{12}$ implica, en uno de sus modos más centrales, la puesta en práctica en el arte de determinadas operaciones formales que den cuenta de una vasta autoconsciencia respecto de la propia limitación, de la imposibilidad del hombre de asir lo absoluto, de abordar el todo. Dice F. Schlegel en sus Ideas: "La ironía es la clara consciencia de la eterna agilidad, de la infinita plenitud del caos" (2009: 204). El mismo Schlegel la había descripto en otro contexto (en los Fragmentos críticos) comentando sus efectos: “Contiene y provoca a la vez un sentimiento del conflicto indisoluble entre lo

\footnotetext{
${ }^{12}$ Rudolf Köpke (1855: 238), en un importante estudio, recupera una declaración de Ludwig Tieck mismo según la cual "Es infinitamente complicado expresar el concepto de ironía en una fórmula determinada. (...) Es lo divino-humano en la poesía. Quien lleve esto consigo como una profundísima convicción y lo haya experimentado, ¿precisará acaso una definición? Esta última solo colocará, al final, una palabra en reemplazo de la otra, y esa quizás se entenderá tan poco como la anterior. En la mayoría de las definiciones la ironía se toma de modo demasiado unilateral, o, podría decir, demasiado prosaico, demasiado material". El tema fue abordado insistentemente desde muy diversas perspectivas. Mencionemos un estudio clásico de muy alto nivel sobre la ironía en el romanticismo (que se ocupa, entre otras cuestiones, de la ironía en la obra dramática de Tieck), Die romantische Ironie in Theorie und Gestaltung, de Ingrid StrohschneiderKohrs (2002 [original de 1960; reeditado con mejoras en 1977]), y una compilación en inglés de estudios sobre la ironía romántica en general hecha por Frederick Garber (1988): Romantic Irony. Los capítulos de Ernst Behler ("The Theory of Irony in German Romanticism") y Raymond Immerwahr ("The Practice of Irony in Early German Romanticism") se ocupan específicamente del caso alemán.
} 
ALBERTI, M. - El Romantismo Alemán y el Drama

condicionado y lo incondicionado, de la imposibilidad y la necesidad de una comunicación completa" (2009: 49). El mundo de los humanos es un mundo de limitaciones, quebrado, y su lenguaje no se basta, en principio, para dar cuenta del mundo libre e ilimitado que se despliega más allá de estos límites: lo mejor que puede hacer es mostrarse consciente de sus falencias, de sus insuficiencias, o, como diría Novalis en una de la más citadas "definiciones" del Romanticismo, potenciar la cualidad de este mundo finito y limitado:

El mundo ha de ser romantizado. Así se reencuentra el sentido original. Romantizar no es sino una potenciación cualitativa. El sí mismo inferior se identifica en esta operación con el sí mismo mejor. Al igual que nosotros mismos somos una cadena cualitativa de potencias de esa especie. Esta operación es aún del todo desconocida. En cuanto doy un sentido elevado a lo vulgar, un porte misterioso a lo habitual, la dignidad de lo desconocido a lo conocido, una apariencia infinita a lo finito, lo romantizo. (1994: 109)

El acto de romantizar es un acto definitivamente no-reproductivo. La "poesía" romántica se piensa, como ya se indicó, como "producción”, e idealmente como producción libre e incondicionada de un Yo creador y espontáneo.

Ahora bien: esta operación clave de "romantización" del mundo es lo contrario de lo que Novalis encontraba en el teatro -al menos en el de su época. Esto a punto tal de decir que "Nuestro teatro es absolutamente no-poético", ya que allí también "tiraniza el principio de la imitación de la naturaleza" (III: 691). En este mismo apunte reclama Novalis para la poesía, con signos de admiración, que sea “¡fantástica!”.

Este es un primer indicio de la distancia que imponía al proyecto romántico el ámbito teatral y sus ya consolidadas aspiraciones a la instrucción del pueblo. En sintonía con la máxima ya mencionada de la imitación de lo bello, el teatro alemán del siglo XVIII había buscado educar mediante la exhibición de casos ejemplificadores, y, sobre todo en la segunda mitad del siglo, mediante la puesta en escena de situaciones de personas más o menos corrientes con las que el auditorio podía identificarse.

El procedimiento de la "poesía" romántica es contrario a este: no expone un caso ejemplar para iluminar al ciudadano común. Más bien envuelve a lo normal en un aire misterioso que lo eleva, favoreciendo así en el lector, oyente, ¿espectador? una ascensión de otro orden que el prodesse moral y prosaico (a ojos de los románticos) del proyecto cultural anterior. Por lo demás, la perspectiva "pedagógica" en general (en el sentido de una intención de educar a la generalidad) era más bien ajena al proyecto del Romanticismo, que se preocupaba mucho más por lo singular que por lo general; que se interesaba mucho más por el "genio" que por el humano corriente; que (al menos en su 
ALBERTI, M. - El Romantismo Alemán y el Drama

primera etapa) tuvo un interés político más bien reducido; que aspiraba mucho más a una elevación metafísica que a un enriquecimiento de los valores ciudadanos. A propósito, no hace falta exagerar el carácter evasivo del primer Romanticismo para conceder que lo tenía en grado sensiblemente más elevado que otros movimientos cercanos en el tiempo (lo que no implica, por supuesto, que lo político fuera algo del todo ajeno a los intereses de los románticos o del todo ausente en sus obras).

En el caso de la ideología ilustrada puede verse con bastante facilidad por qué el escenario era una plataforma adecuada: ¿qué otro vehículo podía ser más directo y eficaz a la hora de inculcar valores o motivos de inspiración a una sociedad, por lo demás, de un altísimo grado de analfabetismo? Pero ¿eran los intereses del Romanticismo tan aptos para las tablas? Un punto sugiere con bastante fuerza que, aun cuando el discurso dramático pudiera atraer a algún autor romántico, eso no implicaba que acabara creando una obra susceptible de ser efectivamente puesta en escena. De hecho, buena parte de esta producción dramática fue escasa o nulamente llevada al escenario, y ello no debido a contingencias, o a preferencias estéticas estéticas del público o la crítica, etc., sino porque muchos de estos dramas románticos no estaban pensados realmente por sus autores para ser presentados en el teatro, como se advierte claramente en los dramas de centenares y centenares de páginas de Brentano y Arnim. Como señala Manfred Schmeling respecto de lo que llama la "paradoja del Romanticismo" en lo que concierne al drama, "La auténtica 'obra de teatro' romántica es, por sobre todas las cosas, meramente un 'fantasma', una creación que no se desarrolla en el escenario (...) sino en la crítica y en la estética" (1994: 35-36). Estos autores escriben un teatro que aspira visiblemente más a ser leído que a ser "asistido": sacan al teatro del teatro.

Planteado el problema desde la perspectiva contraria, y quizás con más precisión: ¿eran las tablas tan aptas para los intereses del Romanticismo? Así formulada la pregunta, seguramente no podrá ser respondida sin incurrir en arbitrariedades; aun así, puede tener valor la cuestión en la medida en que pone a la vista un fenómeno que resulta mucho más mensurable y objetivo: el programa del Romanticismo no solo no acudió al teatro como medio prioritario de expresión y difusión, sino que, además, se ocupó de desarrollar otros discursos que consideraba, explícitamente, más ajustados a su poética. El fragmento, género romántico por excelencia, y la novela, formato privilegiado, sobre todo, por su 
ALBERTI, M. - El Romantismo Alemán y el Drama

capacidad de reunir con naturalidad discursos distintos. ${ }^{13}$ Es decir: por un lado, un género especialmente apropiado para los chispazos de ingenio que expresan de un modo muy ajustado la ironía romántica; y, por el otro, un género versátil en el mayor grado y por esto mismo también uno muy capaz de unificar discursos y de acercarse así lo más posible a una manifestación de la "totalidad" (totalidad a la que se aspira siempre y a la que no se llega nunca). Uwe Japp lo plantea en estos términos:

Es cierto que el proyecto romántico de una presentación de lo infinito en lo finito favoreció una cierta preferencia por la novela -y por la prosa en general-, como por ejemplo en Friedrich Schlegel o Novalis. La amplitud de la forma -junto a una simultánea tendencia a lo fragmentario- parece apropiada para poder dar cuenta, en tanto poesía universal, de la situación de lo individual en el universo. Asimismo, la novela se ajusta a la mezcla de géneros postulada en las premisas de la poesía universal y trascendental. (1999: 2)

Por el contrario, pareciera que el teatro, a los ojos de los románticos, seguía siendo un discurso muy atado a las limitaciones de la realidad, muy atado a la imitación (y, en consecuencia, muy desprovisto de fantasía), demasiado forzado a una ambición pedagógica, demasiado aprisionado por reglas fijas, demasiado marcado. Por otra parte, ¿cómo habría sido una tragedia irónica? ¿Podía una tragedia, como pedía Schlegel (en el "fragmento crítico" ya citado), exponer una mirada en la que "todo debe ser broma y todo debe ser serio"? ¿No se trata de un discurso demasiado definido y seguro de sí como para encarnar esta compleja insuficiencia que se expresa en la ironía romántica? Esto, al menos, parece verosímil de la tragedia, un discurso que correría serios riesgos, capaces de conducirla a su autodestrucción, si se permitiera, por ejemplo, tomarse poco en serio, revelar su carácter de artificio o buscar desautomatizaciones mediante rupturas insistentes de la ilusión escénica. Hay, por cierto, sentidos de la noción de "ironía romántica" que se habrían visto materializados de la manera más perfecta en operaciones "autodestructivas" de esta naturaleza en una tragedia, pero el costo estético que habrían acarreado parece haber sido demasiado alto incluso para la osadía de los románticos tempranos.

El caso de la comedia, sin embargo, es distinto. La comedia sí podía tener la plasticidad necesaria para afrontar este desafío: el desafío de la ironía romántica de mostrar la propia limitación, de revelarse como incompletitud. En todo caso, de los dos discursos, el trágico y el cómico, este último parece ser con mucho el más capaz de subvertir sus propias reglas sin echar a perder su esencia. La comedia resulta mucho más

\footnotetext{
${ }^{13}$ Como indica una célebre cita de Jean Paul, que introduce el parágrafo 69 de su Antesala de la estética (Vorschule der Ästhetik) de 1804, la novela es capaz de reunir, por causa de la amplitud de su propia forma, "casi todas las formas" en general del discurso literario.
} 
ALBERTI, M. - El Romantismo Alemán y el Drama

capaz de ponerse en jaque, de tomarse poco en serio, de mostrarse falible, de, incluso, destruirse a sí misma y lograr precisamente así el efecto deseado. Esto último será desarrollado con más detenimiento en los comentarios siguientes sobre la naturaleza del teatro romántico. En efecto, hablar del teatro romántico, quizás por razones (entre otras) como las recién aludidas, implica en buena medida hablar de comedia romántica.

\section{Elementos del drama romántico}

Poco después de la frase citada en último lugar el mismo Japp señala que a la novela en tanto expresión cumplida de la "poesía universal" tan vinculada al proyecto romántico podría sumársele con derecho un "drama universal" que estaría representado fundamentalmente por las comedias de Ludwig Tieck (1999: 2). Esto introduce uno de los dos ejes centrales de esta sección, dedicada al comentario de algunos aspectos recurrentes en la producción teatral del Romanticismo alemán (aspectos que, como se adelantó en el apartado inicial de este artículo, serán materializaciones dramáticas de una estética romántica general). Este primer eje concierne a la búsqueda de la "reunión de géneros" que, así como es definitoria de la naturaleza de la novela romántica, resulta también característica de su teatro. El segundo eje concierne a una cierta recursividad, que aquí se manifiesta con insistencia, entre otras variantes, en la forma de un "teatro dentro del teatro". Esta recursividad coincide, en esencia, con el proyecto romántico en general de una "poesía de la poesía" (y de una "filosofía de la filosofía") y, como se verá con especial atención, con la propuesta "irónica" de Schlegel.

¿Cuáles son, en primer lugar, los elementos que el "drama universal" del Romanticismo reúne? Una marca que salta a la vista, aunque no resulta en absoluto patrimonio exclusivo del drama romántico, es la reunión de verso y prosa. Este procedimiento de introducción - en principio, ocasional - del verso, que los románticos podían haber tomado ya de sus fuentes más conscientes (como Shakespeare o Calderón), es casi omnipresente desde Tieck. Su variante menos disruptiva, más natural, es la inclusión, en medio del desarrollo de trama, de elementos que en sí mismos ya estaban en verso: mayormente canciones, o en ocasiones también otros discursos, como en Ponce de León de Clemens Brentano la misteriosa profecía de la gitana sobre el final de la obra, etc. Este recurso ya había sido explotado anteriormente en Alemania, y de hecho lo había sido con mucho éxito hacía poco, como, por ejemplo, en el caso de uno de los mayores 
ALBERTI, M. - El Romantismo Alemán y el Drama

hits del escenario alemán de fines del siglo XVIII, Los bandidos de Friedrich Schiller (Die Räuber, 1781), que contiene una de sus poesías más recordadas, allí bajo la forma de una canción (la poesía conocida en general como Hektors Abschied).

En su primer gran drama, El caballero Barba Azul (Ritter Blaubart de 1796), Tieck se sirvió de la misma herramienta expresiva. Sin embargo, ya en El gato con botas (Der gestiefelte Kater, 1797) aparecen versos fuera del contexto de una composición musical y en general sin un marco evidente que le sirva de "justificación”, mecanismo que se potencia en Príncipe Zerbino o el viaje hacia el buen gusto (Prinz Zerbino oder die Reise nach dem guten Geschmack, 1797), en la que verso y prosa alternan en proporciones similares, alternancia que también encontramos en la Vida y muerte de Santa Genoveva (Leben und Tod der heiligen Genoveva, 1799), aquí ya con marcado predominio del verso; en El mundo al revés (Die verkehrte Welt, 1800), la intervención del discurso musical llega al extremo de plantearse el prólogo de la obra expresamente como una "sinfonía"...

Los otros representantes del drama romántico también acudieron con frecuencia al intercambio con el verso. Es el caso, por ejemplo, de Achim von Arnim y Mariano Caboga (aparecida en 1826) o El agujero (Das Loch, en 1813), casi exclusivamente en verso. Ya mencionamos Ponce de León (compuesta en 1801); mencionemos también la otra gran obra teatral de Brentano, La fundación de Praga (Die Gründung Prags, elaborada entre 1812 y 1814), compuesta directamente en verso. Alternancias de diferentes proporciones encontramos también en Joseph von Eichendorff y La guerra a los filisteos (Krieg den Philistern, 1824), Los pretendientes (Die Freier, 1833), etcétera.

No se trata de la única "mezcla" (ni, por cierto, de la más original). Encontramos otros discursos "filtrados" o "intervenidos". En primer lugar, sin salir del ámbito teatral mismo, encontramos entremezcladas, como en el Emperador Octaviano (Kaiser Octavianus, 1804) de Tieck, la comedia y la tragedia (y, de hecho, en este caso, a ambas junto con otros géneros como la novela pastoril o la comedia de enredos). También encontramos la rama aún no mencionada de la tripartición convencional de la "poesía", junto con la lírica y el drama: la épica, de la cual se usan en ocasiones no solo el tono elevado y ciertos tópicos sino también recursos compositivos que le son esencialmente característicos, como por ejemplo el de los repetidos símiles, algunos de los cuales acusan 
ALBERTI, M. - El Romantismo Alemán y el Drama

un evidente origen homérico en lo que a la forma se refiere. ${ }^{14}$ También se asemeja el teatro romántico a la épica en un aspecto formal que le es sumamente propio: su carácter episódico, despojado de una estructura argumental firme, en donde muchas veces cobra mayor visibilidad y protagonismo la pieza que el rompecabezas completo (en ocasiones, por cierto, son precisamente los aspectos extra-argumentales los que constituyen la auténtica obra de teatro, como se señalará sobre el final del artículo). Un rasgo, por lo demás, que no deja de tener un aire de familia con la valoración tan propiamente romántica del fragmento. Esta tendencia a la incorporación de elementos distintivos de otros géneros puede perfectamente sostenerse sin quebrar la obra, pero en cualquier caso parece apuntar a generar una interrupción en la unidad o en la homogeneidad del género dramático.

Encontramos con frecuencia, además, otro discurso entremezclado en las obras de teatro románticas; un discurso muy convocante para el movimiento: el discurso del cuento maravilloso, del Märchen, que aparece no solo en tanto tal sino también como fuente de inspiración para los temas en general de las obras. Casos evidentes de esto último, entre otros, son las obras de Tieck El gato con botas o Vida y muerte de la pequeña Caperucita Roja (Leben und Tod des kleinen Rotkäppchens, 1800). El Märchen se ajustaba a la perfección al rol de fuente e interlocutor para un teatro que reclamaba, como en el pasaje arriba mencionado de Novalis, un aumento en el grado de fantasía. Esta apelación a la forma y el material del mundo maravilloso del Märchen significa un fuerte corrimiento respecto del contenido elegido por los dramaturgos de los períodos inmediatamente anteriores de la historia del drama alemán: la inspiración, en cuanto a la temática, ya no parte ni de la realidad de la familia burguesa de la época ni de la idealidad de las antiguas leyendas griegas y romanas, sino que, por un lado, se apunta, en la medida en que el apoyo ya no proviene de la realidad sino de la fantasía, en una dirección más libre e innovadora, $y$, por el otro, en la medida en que las fuentes de las que se abreva no se encuentran en la literatura clásica sino en la tradición popular europea en general o germánica en particular, se reformulan las filiaciones y se establecen vínculos que no solo tienen interés en términos estéticos. El Märchen, de hecho, como mundo de historias, cosmovisiones y símbolos, le aportaba también al teatro (tanto como a la narrativa) un lazo con la tradición

\footnotetext{
${ }^{14}$ Véase por ejemplo el siguiente, de la Genoveva de Tieck: "Así como un ojo en la noche, como un orificio, / en que uno ve la mañana a través de una negra cortina, / y como luego todo se apaga y se hunde en sí, / primero el fulgor, luego el carbón, y por último / la sorda ceniza: así es también la vida del guerrero; (...)" (1963: 449).
} 
ALBERTI, M. - El Romantismo Alemán y el Drama

y con lo nacional que irá tornándose cada vez más presente en tanto objeto de estudio y fuente de inspiración conforme avancen los primeros años y las primeras décadas del siglo XIX alemán y conforme despierte el brote nacionalista que se generó (o se exacerbó marcadamente) con la dominación napoleónica. Este interés por lo nacional no se juega solamente en el plano "mitológico" del cuento maravilloso, sino que está muy presente, en el drama romántico, también en un sentido histórico (tendencia que ya se había manifestado o se iba manifestando en varias obras de otros autores: particularmente en Schiller; o en algunas obras de Goethe, como por ejemplo en el ya mencionado Götz von Berlichingen).

El teatro romántico (siguiendo los pasos del drama del Sturm und Drang, pero con giros propios) abordó, cada vez más, temas históricos nacionales, aspecto especialmente visible en la obra dramática de Friedrich de la Motte Fouqué, quien, de hecho, no solo tomó temas históricos nacionales sino también cuestiones políticas de actualidad (cf. STOCKINGER 2004: 136-137). Se trataba de un interés que había sido estimulado más de una vez desde otro escenario, distinto del teatral pero que en la época funcionó como plataforma para parejas explosiones de oratoria y desgarramiento: el del profesor universitario. Piénsese, en términos generales, en la arenga inicial (hecha muy a fines de 1807) de los Discursos a la nación alemana de Fichte (1984); o, sobre todo, en el cierre -referido específicamente a la misión política del teatro- de las clases de August Wilhelm Schlegel de 1808 (publicadas entre 1809 y 1811). Es indicio de un aire de época que A.W. Schlegel, uno de los fundadores del Romanticismo temprano (grupo mucho menos politizado, en una primera instancia, que el del "alto Romanticismo" posterior), haya hecho en 1808, es decir, unos pocos años después del declive del mayor período creativo del grupo, un llamamiento expreso y enfático al fortalecimiento de un teatro nacional, en la última de estas Lecciones sobre arte dramático y literatura, en términos de un nacionalismo bastante desatado. Un nacionalismo que reclama volver a rescatar lo que "los alemanes fueron antiguamente y han de volver a ser", que llama la atención sobre el "peligro" en que se encuentran los alemanes de "desaparecer completamente de la serie de países autónomos" al insistir en una nociva "preferencia por las costumbres extranjeras". ¡Justamente "nosotros, antaño el primero y el más glorioso pueblo de Europa"!...

Una mención aparte merece, en este punto, la obra dramática de Fouqué, tanto por cuestiones relativas a su contenido, frecuentemente ligado a la historia de Alemania y a 
ALBERTI, M. - El Romantismo Alemán y el Drama

su tradición literaria y mitológica, como por cuestiones referidas a la forma. Respecto de esto último, Fouqué logró, en sus dramas, retomar de la tradición romántica alemana el programa de Schlegel de una obra de arte que armonice géneros y discursos distintos, integrando con particular intensidad tradiciones artísticas musicales, con lo que a la vez proveyó a la continuación del Romanticismo un modelo para lo que posteriormente sería el proyecto wagneriano de una obra de arte total. ${ }^{15}$ Facilitó de este modo un puente entre una de las primeras formulaciones programáticas del movimiento y una de las últimas manifestaciones innovadoras de Romanticismo en el escenario. Al recientemente mencionado Fichte, precisamente, dedica la poesía que introduce la primera parte de su gran drama El héroe del Norte (Der Held des Norden, 1808-1810), en donde recogió de la tradición literaria nacional la historia de los Nibelungos, que Wagner reformularía, apoyándose en Fouqué, entre 1848 y 1874, en su tetralogía operística. ${ }^{16}$ En general encontramos en Fouqué un interés marcado tanto por cuestiones históricas como por tradiciones mitológicas germánicas, a las que remitió con insistencia y fidelidad en su obra. También su obra más difundida entre lectores de habla hispana Ondina (Undine, 1811), en este caso un relato, aborda materia mitológica germánica.

Tratamos sobre incorporaciones de otros discursos literarios y determinadas temáticas en el teatro; también podría señalarse, a la inversa, la incorporación del teatro en otros discursos. En particular, en las novelas, en las que se insertaban ocasionalmente escenas o incluso desarrollos teatrales complejos. Pero hay, sobre todo, una incorporación que reúne, podría decirse, ambos lados de la interacción del teatro con otros registros literarios (la intervención del teatro en otros textos y la incorporación de otros discursos en el teatro) en un formato particularmente caro al drama romántico: la incorporación del teatro en el teatro.

Las prácticas de "metateatro", como se lo bautizara en el clásico de Lionel Abel Metatheatre: a new view of dramatic form (1963), ${ }^{17}$ aunque muy lejos de ser una innovación del drama romántico, sí son uno de sus rasgos más salientes; uno de los que

\footnotetext{
${ }^{15}$ Claudia Stockinger se ocupa, en la sección final (precisamente, "Gesamtkunstwerk") de su trabajo dedicado íntegramente a la obra dramática de Fouqué (2000), de la significativa integración, en las obras teatrales de Fouqué, de distintas tradiciones musicales nacionales y europeas.

${ }^{16}$ Para los vínculos entre la tetralogía de Wagner y Der Held des Norden de Fouqué véase el artículo de W.G. Schmidt (2001) "Der ungenannte Quellentext. Zur Wirkung von Fouqués Held des Nordens auf Wagners Ring-Tetralogie". La tesis del mismo Schmidt (2000) consiste en un estudio del Héroe del Norte en general.

${ }^{17}$ En el texto de Larson (1992) mencionado en las "Referencias bibliográficas" se encuentra una clara exposición crítica en español de este concepto y de ciertas nociones vecinas.
}

Pandaemonium, São Paulo, v. 25, n. 45, jan.- abr. 2022, p. 110-135 
ALBERTI, M. - El Romantismo Alemán y el Drama

más le dan unidad al "teatro romántico" en tanto tal, y esto no solo respecto del drama alemán sino en general del del Romanticismo como un todo (cf. SCHMELING 1994: 36).

No debería llamar la atención si recordamos que se trata de un movimiento que, entre otro de sus aspectos más definitorios (y en este caso particularmente distintivo) tiene el de unificar programáticamente la teoría con la práctica, la reflexión con la poesía. Buena parte del proyecto romántico se expresa y se ejecuta al mismo tiempo en el plano de lo "meta-". Poesía sobre la poesía, filosofía sobre la filosofía, lógos sobre el lógos (lo que Novalis llamaría "logología”), teatro sobre el teatro: todas estas manifestaciones están en sintonía con el proyecto general de la "poesía universal progresiva" de la que hablaba Schlegel como la propia del Romanticismo. Es una "poesía" que debe poder unificar no solamente los distintos géneros literarios; ni siquiera los distintos géneros discursivos en general; debe incluso proyectarse sobre el mundo extra-literario de "la vida y la sociedad":

La poesía romántica es una poesía universal progresiva. Su destino no es sólo reunir los géneros separados de la poesía y poner en contacto la poesía con la filosofía y la retórica; también quiere, e incluso debe, ora mezclar, ora fundir poesía y prosa, genialidad y crítica, poesía artificial y poesía natural, hacer más viva y social la poesía, más poética la vida y la sociedad; debe poetizar el ingenio, y colmar y saturar las formas del arte con las más puras y más variadas materias de la cultura, animándolas con las pulsaciones del humor. (2009: 81)

La reflexión sobre el teatro en el contexto del teatro mismo habría sido un imperativo romántico aún si no hubiera existido una tradición metateatral en la que apoyarse. De hecho, no resulta casual sino más bien sintomático que los principales modelos a los que remiten los románticos sean Calderón, Cervantes y Shakespeare. En un sentido amplio, la parábasis, en la que la dinámica propia de la obra se aparta o se suspende para introducir y proponer al lector o espectador una reflexión sobre la obra misma, forma parte del núcleo de la poética romántica y se ejecuta de manera expresa o más o menos disimulada en una multitud de obras románticas (del género que sean). A esta reflexión sobre la obra en la obra misma; a esta auto-exposición y auto-justificación del teatro romántico se la ha entendido como una proyección del mismo programa que expresamente defendían los primeros románticos que hacían teoría sobre su práctica, Friedrich Schegel y Novalis, bajo la denominación de "Transzendentalpoesie”, "poesía 
ALBERTI, M. - El Romantismo Alemán y el Drama

trascendental". De allí que se hable, en este caso, de "Transzendentaldrama", "drama trascendental". 18

Las operaciones metateatrales de los románticos, por lo demás, entroncan de manera muy espontánea con la puesta en práctica de la "ironía". ${ }^{19}$ Benjamin afirmó, en su tesis sobre El concepto de crítica de arte en el Romanticismo alemán, que el drama es en general el ámbito más propicio para un uso eficaz de la ironía:

La ironización de la forma consiste en su destrucción voluntaria, según evidencian en la forma más extrema, entre las producciones románticas y probablemente en toda la literatura en general, las comedias de Tieck. De entre todas, es la forma dramática la que puede ser ironizada en más alta medida y de la manera más profunda y eficaz puesto que contiene la más alta medida de fuerza de ilusión y, merced a ello, puede absorber un elevado grado de ironía sin disolverse completamente. (2002: 93)

El drama en general, o, más bien, como ya se adelantó, parece ser la comedia en particular el marco más ajustado para esta ironía. La comedia tiene una capacidad sobresaliente para procesar operaciones autodestructivas sin colapsar; es más: hay, en sus vueltas sobre sí misma, en sus puestas en evidencia de sus propios recursos, una operación que contribuye con el éxito mismo de su empresa, la generación de un efecto cómico. Es el efecto que se produce cuando, en el Príncipe Zerbino, interviene el autor para evitar que el actor escape de la obra para esquivar el destino que se le asignó en el libreto; o cuando un personaje reclama en el acto cuatro que se le tenga paciencia, que se le deje llegar al final de la obra para así poder reivindicarse en el quinto. O cuando, en El gato con botas, los espectadores conversan entre sí (¡y con el autor!) sobre la obra, o cuando el gato defiende la necesidad de una acción "por el bien del público" (ya que de no realizarse esta acción "la obra no termina más"). O cuando el público y los actores intercambian roles en El mundo al revés. Y sobre todo en los desenlaces -o, mejor dicho, en la falta de auténtico desenlace característica de la comedia romántica- la ironía se muestra en su formato más exitoso.

La realización máxima a la que aspira El gato con botas es, precisamente, la exhibición de su fracaso. Una vez que la obra se clausura, tras llegar de un modo completamente accidentado y deficitario al presunto cierre del argumento (del argumento de la obra dentro de la obra), ya con el decorado retirado y las paredes del teatro al descubierto, aparece el autor para sostener un breve diálogo con sus críticos Fischer,

\footnotetext{
${ }^{18}$ Ya Bernhard Heimrich (1968) y mucho más recientemente Scherer, que se ocupa en detalle del asunto (2003: 94 ss.).

${ }^{19}$ Está dedicado a estos vínculos entre "el metadrama, la obra dentro de la obra y la ironía romántica" todo un capítulo del libro de Ruth Petzoldt (2000: 73-94) Albernheit Mit Hintersinn.
}

Pandaemonium, São Paulo, v. 25, n. 45, jan.- abr. 2022, p. 110-135 
ALBERTI, M. - El Romantismo Alemán y el Drama

Müller y Schlosser, a los que acusa de no haber colaborado con el éxito de la pieza: su objetivo era que ellos se despojaran de sus aprendizajes y lecturas y que disfrutaran con la sensibilidad espontánea que tenían de niños, a lo que le responden “¡Pero gracias a Dios que ya no lo somos!”; “ßBastante esfuerzo y sudor frío nos costó nuestra formación!”. En un último intento, el autor se dirige al público en general aspirando a su comprensión y recibe a cambio bolas de papel y frutas podridas. El cierre, apenas después, es un reclamo al "siglo desagradecido" que recuerda el lamento final, en un contexto infinitamente más solemne, de las últimas líneas de Götz von Berlichingen. Más extremo aún es el caso de la composición dramática en verso Un prólogo (Ein Prolog, 1797), en donde el telón nunca se abre y todo gira en torno a una obra de teatro que nunca comienza. En un gesto que pinta su ironía de cuerpo entero, Tieck hace de la inexistencia de la obra una obra en sí misma.

\section{Recapitulación}

Schmeling afirma que "La práctica teatral romántica es, en el mejor de los casos, solo una aproximación a los conceptos motivadores de la poética romántica" (1994: 36). La frase puede despertar cierta antipatía, porque parece suponer una crítica (en cuyo caso bastante controversial) del drama romántico a partir de su dependencia de la teoría romántica en general. Pero bien podría aceptarse sin incomodidad, parafraseando a Schmeling, que "la práctica teatral romántica es, merced a que se trata de uno de los movimientos con mayor autoconsciencia y más alto grado de interés en la vinculación entre teoría y práctica, una plasmación dramática de los conceptos motivadores de la poética romántica”. De cualquier movimiento cabría esperar que dejara ver cierta "coherencia" (sin matiz valorativo) entre sus ideas sobre el arte y la realización de tales ideas en obras de arte concretas. Es de suponer que un movimiento que ubicó sus desarrollos teóricos en una posición tan visible (a veces predominante) muestre un entrelazamiento más marcado entre obra y poética que el que se observa en otros casos. En ocasiones, incluso, un entrelazamiento forzado. O uno particularmente exitoso en otras. F. Schlegel supo ofrecer ejemplos claros de lo primero. El de Tieck podría ser, repetidamente, un caso de lo último. De todos modos, sin entrar en una cuestión que en última instancia pertenece con más pleno derecho al ámbito del juicio estético personal, señalemos cuáles son algunos de estos aspectos propios de la poética romántica que aquí indicamos como propios de su 
ALBERTI, M. - El Romantismo Alemán y el Drama

producción teatral (ya sea que esto obedezca a un filtrado forzado de las ideas en la práctica o a una sintonía más espontánea entre pensamiento y obra).

Lo primero que aquí se señaló sobre los vínculos del Romanticismo alemán con el teatro trató sobre un parcial distanciamiento del movimiento respecto de la composición de dramas y, aún más, de su representación. Hay una rama del espíritu romántico alemán que no se ajusta con tanta naturalidad a las tablas como a la narrativa; hay algo reconcentrado, íntimo, meditado y desprovisto de acción que el teatro podía solo malamente exhibir; hay algo particularmente fantaseoso, también, que en el teatro podía tender a mostrarse más como una degradación "infantil”" que como la "potenciación" que debía suponer; por lo demás, el Romanticismo sabe que juega, desde el comienzo, con lo inefable, con lo que no puede ser sino aludido, bordeado o señalado. Podemos pensar que había algo demasiado visible en el escenario, demasiado poco alusivo. De todos modos, estas indicaciones pueden ayudar a entender el relativo alejamiento del teatro de parte de los románticos; y fracasan estrepitosamente, en cambio, cuando consideramos que sí existe de hecho (aunque no fuera la primera línea del movimiento) un drama romántico que (lo que es más importante) también exhibe una notoria hermandad con las pulsiones generales del espíritu romántico y sus plasmaciones teóricas. Aquí se repasaron dos cuestiones que son tan definitorias del teatro romántico como del movimiento en general. Por un lado, su búsqueda de ejecutar, mediante un teatro que absorbe las formas y materiales de los restantes discursos literarios, un "drama universal" en consonancia clara con el programa de una "poesía universal". Por el otro lado, la particular versatilidad del teatro romántico (en particular, la comedia) para vehiculizar la ironía y ponerla en acción.

\section{Referencias bibliográficas}

ABEL, Lionel. Metatheatre: a new view of dramatic form. Nueva York: Hill and Wang, 1963. ARNALDO, Javier (ed.). Fragmentos para una teoría romántica del arte. Madrid: Tecnos, 1994. ARNIM, Ludwig Achim von. Sämtliche Romane und Erzählungen. Múnich: Hanser, 1962.

ARNIM, Ludwig Achim von. Das Loch. In: Kluge, Gerhard (ed.). Das Loch oder Das wiedergefundene Paradies. Das Incognito oder Die mehreren Könige oder Alt und Neu. Achim von Arnim und Joseph von Eichendorff. Berlín: de Gruyter, 1968, p. 5-34.

BEHLER, Ernst. The Theory of Irony in German Romanticism. In: GARBER, Frederick (ed.). Romantic Irony. Ámsterdam: John Benjamin, 1988, 43-81.

BENJAMIN, Walter. El concepto de crítica de arte en el Romanticismo alemán. Madrid: Editora Nacional, 2002.

Pandaemonium, São Paulo, v. 25, n. 45, jan.- abr. 2022, p. 110-135 
ALBERTI, M. - El Romantismo Alemán y el Drama

BREnTANO, Clemens. Sämtliche Werke und Briefe (Historisch-kritische Ausgabe). Stuttgart: Kohlhammer, 1975.

COMETA, Michele. Die Theorie des romantischen Dramas bei Friedrich Schlegel. In: JAPP, Uwe; SCHERER, Stefan; STOCKINGER, Claudia (ed.). Das romantische Drama. Produktive Synthese zwischen Tradition und Innovation. Tübingen: Max Niemeyer, 2000, 21-43.

EICHENDORFF, Joseph von. Sämtliche Werke (Historisch-kritische Ausgabe). Tübingen: Max Niemeyer, 1962.

ENDRES, Johannes. Szenen der „Verwandlung“. Novalis und das Drama. In: JAPP, Uwe; SCHERER, Stefan y STOCKINGER, Claudia (ed.). Das romantische Drama. Produktive Synthese zwischen Tradition und Innovation. Tübingen: Max Niemeyer, 2000, 65-87.

FICHTE, Johann Gottlieb. Discursos a la nación alemana. Barcelona: Orbis, 1984.

GARBER, Frederick (ed.). Romantic Irony. Ámsterdam: John Benjamin, 1988.

GOETHE, Johann Wolfgang von. Werke. Briefe, Tagebücher und Gespräche. Frankfurter Ausgabe in 40 Bänden. Frankfurt am Main: Deutscher Klassiker Verlag, 1985.

GOETHE, Johann Wolfgang von. Götz de Berlichingen, el de la mano de hierro. In: Obras completas: Tomo I. Madrid: Aguilar, 1957.

GoETHE, Johann Wolfgang von. En el día de Shakespeare. In: Ensayos sobre arte y literatura. Málaga: Analecta Malacitana, 2000, 137-141.

GotTSCHED, Johann Christoph. Ausgewählte Werke. Berlín: De Gruyter, 1968.

GROVAS, Víctor. El mundo al revés y la sonrisa romántica. Un viaje por la comedia de Ludwig Tieck. México: Facultad de Filosofía y Letras UNAM, 2001.

HEINE, Heinrich. Die romantische Schule. In: Werke und Briefe in zehn Bänden, 5: Die romantische Schule. Berlín: Aufbau, 1961.

HINDERER, Walter (ed.). Goethe und das Zeitalter der Romantik. Würzburg: Könishausen \& Neumann, 2002.

IMMERWAHR, Raymond. The Practice of Irony in Early German Romanticism. In: GARBER, F. (ed.). Romantic Irony. Ámsterdam: John Benjamin, 1988.

JAPP, Uwe. Die Komödie der Romantik. Typologie und Überblick. Berlín: de Gruyter, 1999.

JAPP, Uwe; SCHERER, Stefan; STOCKINGER, Claudia (ed.). Das romantische Drama. Produktive Synthese zwischen Tradition und Innovation. Tübingen: Max Niemeyer, 2000.

PAUL, Jean. Sämtliche Werke. Múnich: Carl Hanser, 1959.

PAUL, Jean. Antesala de la estética y Sobre la magia natural de la imaginación. México: Ítaca, 2012.

KLUCKHOHN, Paul. Das Ideengut der deutschen Romantik. 2. ed. Halle: Niemeyer, 1942.

KREMER, Detlef. Prosa der Romantik. Stuttgart: Metzler, 1997.

KREMER, Detlef; KILCHER, Andreas B. Romantik. Stuttgart: Metzler, 2015.

KÖPKE, Rudolf. Ludwig Tieck: Erinnerungen aus dem Leben des Dichters nach dessen mündlichen und schriftlichen Mittheilungen. Vol. 2. Leipzig: Brockhaus, 1855.

LARSON, Catherine. El metateatro, la comedia y la crítica: Hacia una nueva interpretación. In: VILANOVA, Antonio (ed). CONGRESO DE LA ASOCIACIÓN INTERNACIONAL DE HISPANISTAS, X, 1989, Barcelona. Actas. Barcelona: Promociones y Publicaciones Universitarias, 1992, 1013-1019.

LOVEJOY, Arthur. On the Discrimination of Romanticisms. Publications of the Modern Language Association of America, v. 39, n. 2, 229-253, 1924.

MÜller-Michaels, Harro (ed.). Deutsche Dramen. Interpretationen. Band 1: Von Lessing bis Grillparzer. Königstein Im Taunus: Athenäum, 1985.

Musset, Alfred de. Lettres de Dupuis et Cotonet. In: Oeuvres Complètes, Tome 9. París: Charpentier, 1888.

Pandaemonium, São Paulo, v. 25, n. 45, jan.- abr. 2022, p. 110-135 
ALBERTI, M. - El Romantismo Alemán y el Drama

Novalis. Schriften. Die Werke Friedrich von Hardenbergs (Historisch-kritische Ausgabe). Stuttgart: Kohlhammer, 1960.

Novalis. Estudios sobre Fichte y otros escritos. Madrid: Akal, 2007.

PETZOLDT, Ruth. Albernheit mit Hintersinn. Intertextuelle Spiele in Ludwig Tiecks romantischen Komödien. Würzburg: Könighausen und Neumann, 2000.

LANGBEHN Regula Rohland de; VEDDA, Miguel (ed.). La teoría del drama en Alemania (17301850). Madrid: Gredos, 2004.

SAFRANSKI, Rüdiger. Romantik. Eine deutsche Affäre. München: Carl Hansen, 2007.

SCHANZE, Helmut. Erfindung der Romantik. Stuttgart: Metzler, 2018.

SCHERER, Stefan. Witzige Spielgemälde. Tieck und das Drama der Romantik. Berlín: de Gruyter, 2003.

SCHILLER, Johann Christoph Friedrich. Sämtliche Werke (Horenausgabe). München: Carl Hanser, 1910.

SCHILLER, Johann Christoph Friedrich. Los bandidos. Madrid: Cátedra, 2006.

SCHLEGEL, August Wilhelm. Sämtliche Werke. Hildesheim: Olms, 1971.

SCHLEGEL, Friedrich. Kritische Friedrich-Schlegel-Ausgabe. Paderborn: Schöningh, 1958.

SCHLEGEL, Friedrich. Lucinda. México: Siglo XXI, 2007.

SCHLEGEL, Friedrich. Fragmentos, seguidos de Sobre la incomprensibilidad. Barcelona: Marbot, 2009.

SCHMELING, Manfred. "Theater in the Theater" and "World Theater". Play Thematics and the Breakthrough of Romantic Drama. In: GILLESPIE, Gerald (ed.). Romantic Drama. Ámsterdam: John Benjamin, 1994, 35-58.

SCHMIDT, Wolf Gerhard. Friedrich de la Motte Fouqués Nibelungentrilogie „Der Held des Nordens “. Studien zu Stoff, Struktur und Rezeption. Röhrig: Universitätsverlag, 2000.

SCHMIDT, Wolf Gerhard. Der ungenannte Quellentext. Zur Wirkung von Fouqués Held des Nordens auf Wagners Ring-Tetralogie. Athenaeum, v. 11, 159-191, 2001.

SCHULZ, Gerhard. Die deutsche Literatur zwischen Französischer Revolution und Restauration. Teil 1. Das Zeitalter der Französischen Revolution: 1789-1806. München: Beck, 1983.

SCHULZ, Gerhard. Romantisches Drama. Befragung eines Begriffes. In: JAPP, Uwe; SCHERER, Stefan; Stockinger, Claudia (ed.). Das romantische Drama. Produktive Synthese zwischen Tradition und Innovation. Tübingen: Max Niemeyer, 2000, 1-19.

StOCKINGER, Claudia. Das dramatische Werk Friedrich de la Motte Fouqués: Ein Beitrag zur Geschichte des romantischen Dramas. Berlín: de Gruyter, 2000.

StOcKINGER, Claudia. The Romantic Drama: Tieck, Brentano, Arnim, Fouqué, and Eichendorff. In: MAHONEY, Dennis F. (ed.). The Literature of German Romanticism. Rochester: Camden House, 2004, 125-145.

STROHSCHNEIDER-KoHRS, Ingrid. Die romantische Ironie in Theorie und Gestaltung. 2. ed. Tübingen: Max Niemeyer, 2002.

TIECK, Ludwig. Werke in vier Bänden. Múnich: Winkler, 1963.

WELLEK, René. The Concept of Romanticism in Literary History. Comparative Literature, v. 1, n. 2, 147-172, 1949.

WIESE, Benno von (ed.). Das deutsche Drama. Vom Barock bis zur Gegenwart. Interpretationen. I. Düsseldorf: Bagel, 1968. 